\title{
Combining temporal relations and signal processing into Interactive Scores
}

\author{
Mauricio Toro \\ Universidad Eafit
}

October 4, 2018

\section{Introduction}

Process calculi has been applied to the modeling of interactive music systems [18, 17, 23, 28, 16, 27, 1, 24, 19, 4, 14, 11, 13, 15, 2, 22, 12, 20, 21, 10] and ecological systems [25, 6, 26, 7]. In addition, research on algorithms [5, 3, 9] and software engineering [8] also contributes to this field.

\subsection{1 table for faust}

\subsection{Block diagram algebra}

\subsection{Example 1}

\subsubsection{Block-diagram algebra for Figure 3 ,}

Acquisition $:=$ Difussion $:=$

Process :=((Acquisition : @ $\Delta_{1}$ : Treatment : Difussion $)$, Mp3 $):>$ _

\subsubsection{Ntcc model}

LowFrec $_{I, S, P r, \mathscr{R}}={ }_{\text {def }}$ Points $_{I, S, P r} \|$ Intervals $_{\mathscr{R}} \|$ User $_{I}$

$\|\operatorname{Clock}(0)\| \prod_{i \in S \cup I} ! \operatorname{tell}\left(0 \leq p_{i}<n_{\infty}\right)$

HighFrec $_{\text {input }, \text { output }}={ }^{\text {def }}$ Acquisition(input,$\left.x\right)\left\|@ \Delta_{1}(x, y)\right\|$ Treatment $(z, w)$

$\|\operatorname{Difussion}(w, u, v)\| M p 3(!, q) \| !$ tell $($ out put $=u+v+q)$

System $_{I, S, P r, \mathscr{R}}={ }^{\text {def }}$ HighFrec $_{I, S, P r, \mathscr{R}} \|$ LowFrec $_{\text {input }, \text { out put }}$

\subsection{Example 2}

\subsubsection{Block-diagram algebra for Figure 8 .}

Acquisition :=

Process := ((Acquisition : Arpeggio), $\mathrm{Mp} 3):>$ 


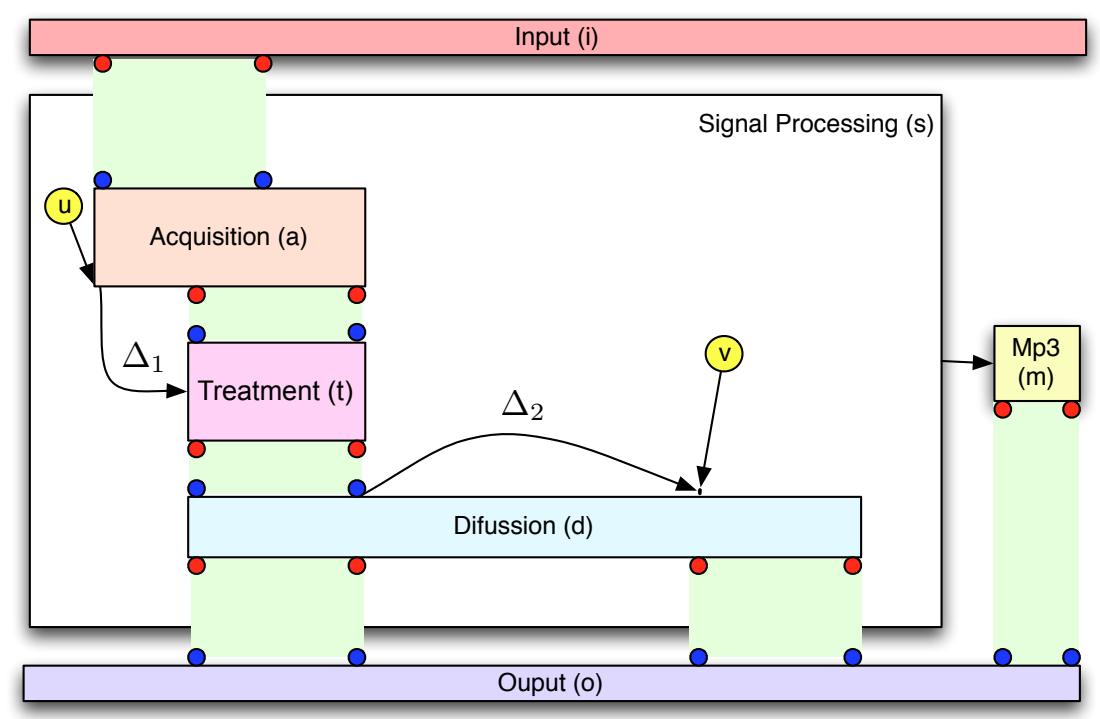

Figure 1: Example of Interactive Score

$\Delta_{i}$

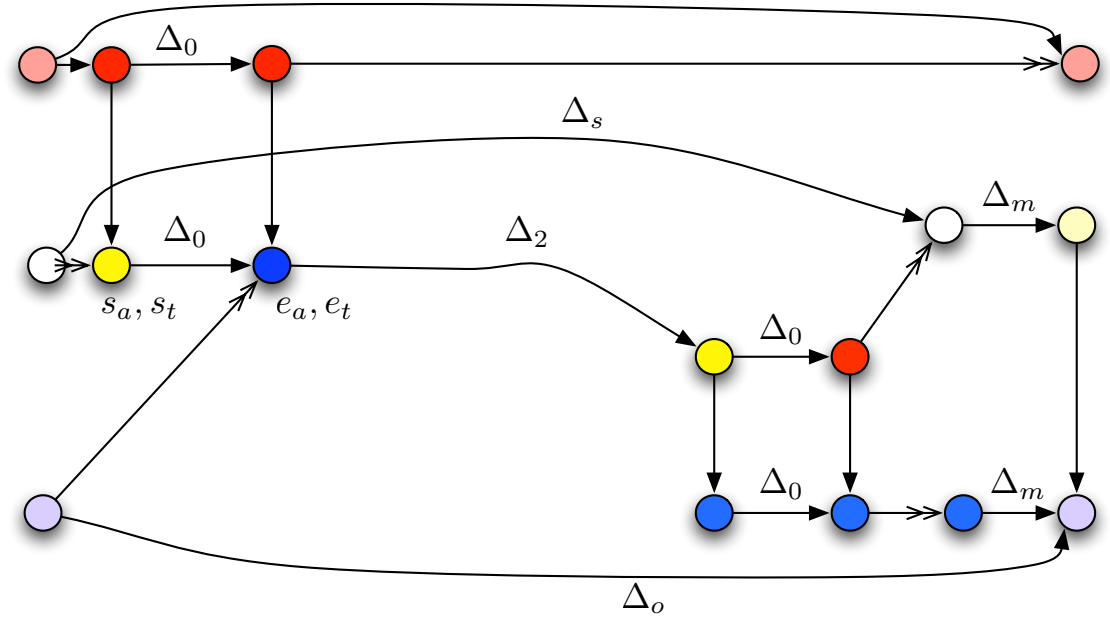

Figure 2: Example of the Event Structure semantics of the Interactive Score in Figure ?. We suppose that $\Delta_{1}=0$. 


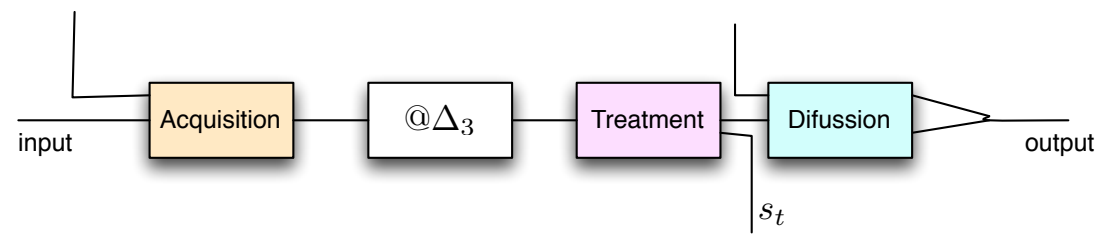

Figure 3: Example of the block diagram semantics of the Interactive Score in Figure $1 . \Delta_{1}$ must be a unary set.

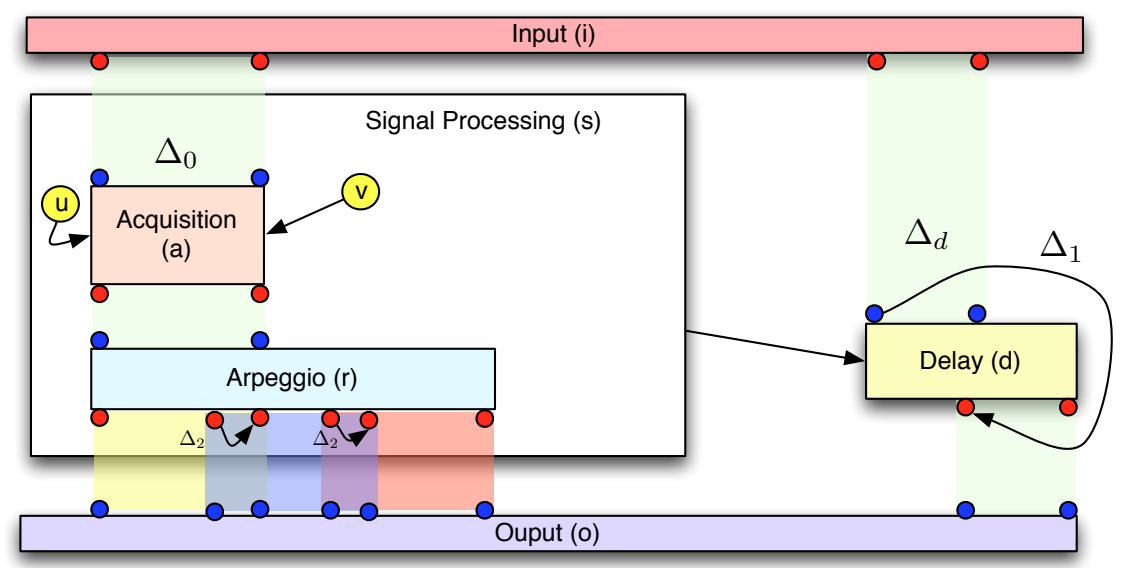

Figure 4: Example of Interactive Score 
$\Delta_{i}$

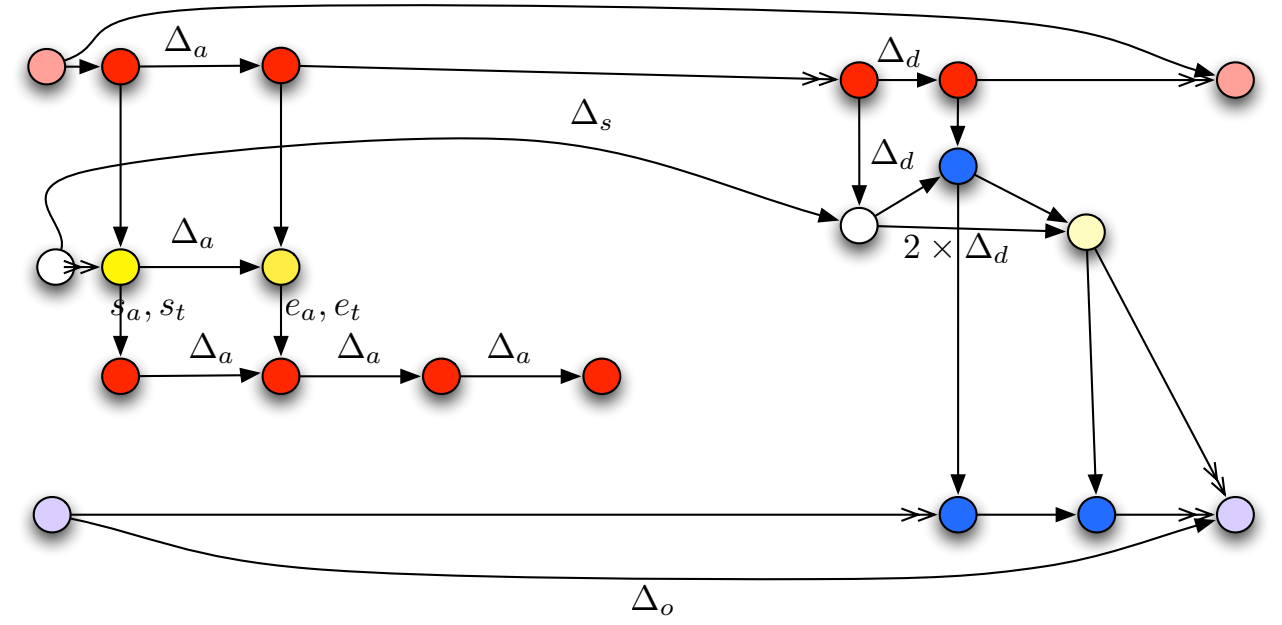

Figure 5: Example of the Event Structure semantics of the Interactive Score in Figure ?. We suppose that $\Delta_{1}=0$.

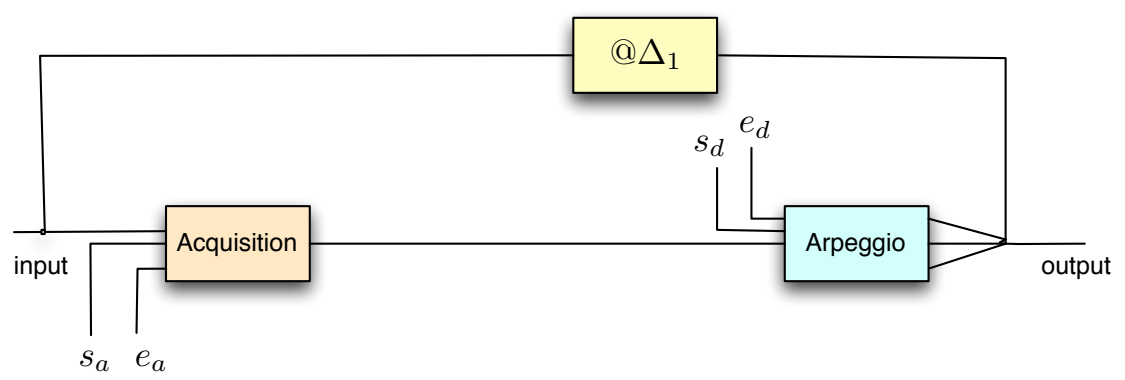

Figure 6: Example of the block diagram semantics of the Interactive Score in Figure $1 . \Delta_{1}$ must be a unary set. 


\begin{tabular}{|ll|}
\hline Signals & Meaning \\
\hline Constant signals & Always same value \\
Integer signals & Always same integer \\
Tuples of signals & n-tuples of signals \\
\hline \hline Signal Processors (S.P) & Meaning \\
\hline Identity box & Identity function \\
Cut box & one input, no output \\
\hline \hline S.P. composition & Symbol \\
\hline Sequential & $:$ \\
Parallel & $;$ \\
Split & $<:$ \\
Merge & $:>$ \\
Recursive & $\sim$ \\
\hline \hline Faust primitives & Examples \\
\hline Arithmetic &,,$+- \times, /$ \\
Comparison & $<, \leq,>, \geq,=$ \\
Bitwise & $<<,>>, \&, \mid$ \\
Constants & $1,2,3,4$ \\
Read Tables & read only buffer \\
Read and write table & read\&write buffer \\
Selectors & conditional branching \\
Graphical interface & buttons, checkboxes \\
\hline
\end{tabular}

Table 1: Semantics of Faust.

\section{Faust}

A signal is a function $s: \mathbb{N} \longrightarrow \mathbb{R}, s(t)$ means signal at discrete time $t \in \mathbb{N}$. Set $\mathbb{S}$ is the set of all signals. A signal processor is a function from tuples of signals to tuple of signals $p: \mathbb{S}^{n} \longrightarrow \mathbb{S}^{m}$.

\section{Complete framework}

\section{Encoding}

\subsection{Encoding a signal}

$\llbracket s \rrbracket_{n}=\prod_{i \in[0, n]}$ next $^{i}$ tell $(s=\mathrm{s}(i))$ 

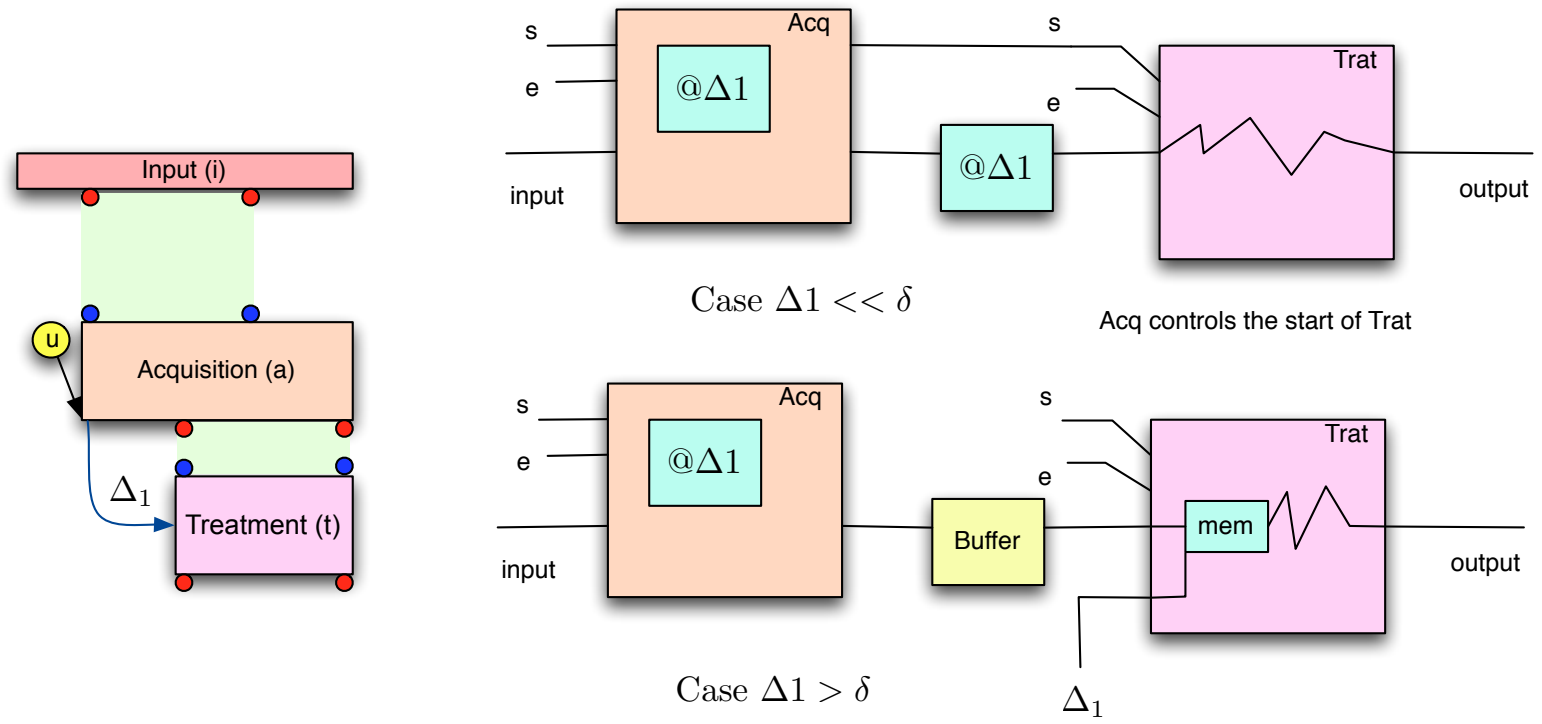

Acq save its output in a Buffer and Trat read it respecting the delay

Figure 7: Strategies to represent macroscopic and microscopic delays

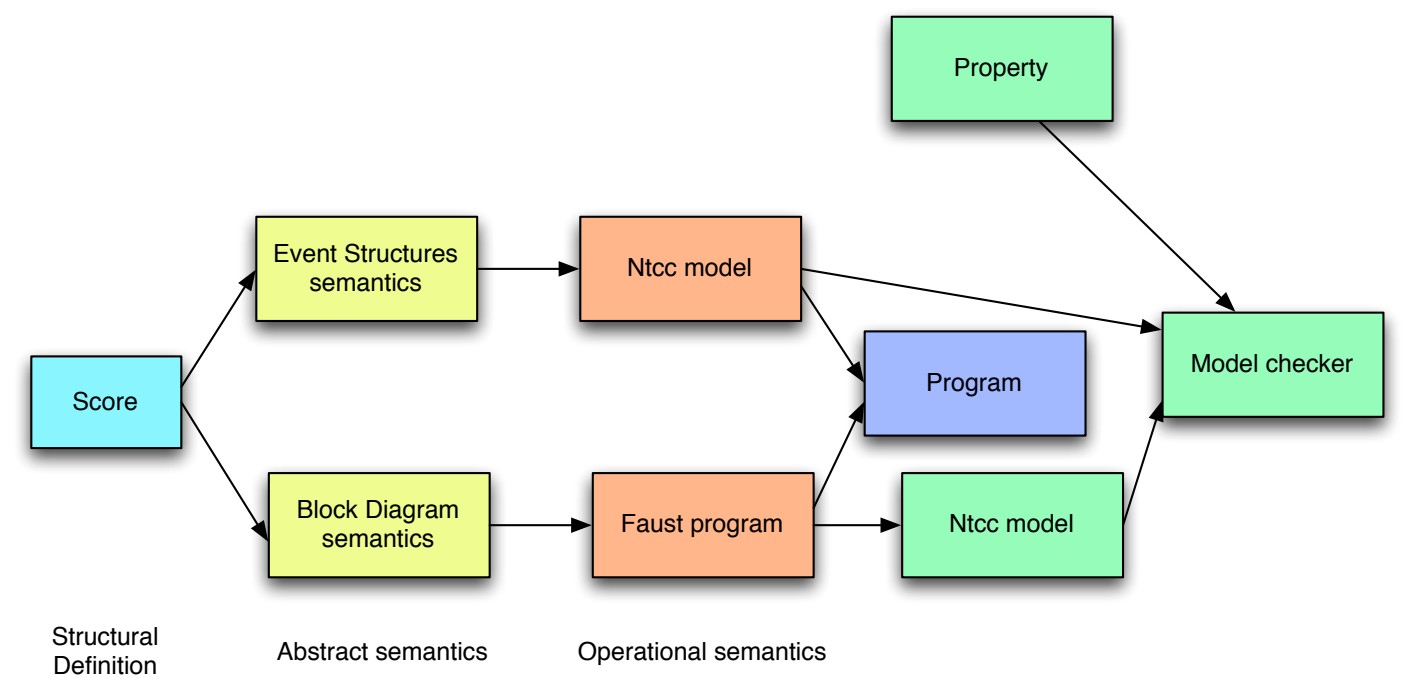

Figure 8: ? 


\subsection{Encoding primitive signal processors}

We assume the existence of the constraints for arithmetic and bitwise operators $* \in\{+,-, \times, /, \%\} \cup\{<<,>>, \&, \mid, \wedge\}$ and for comparison operators $\bowtie \in\{<,>$ $, \leq, \geq, !=,==\}$ in the constraint system.

$$
\begin{aligned}
& \llbracket * \rrbracket_{n}\left(\text { in }_{1}, \text { in }_{2}, o u t_{1}\right)=\text { !tell }\left(o u t_{1}=o p_{1} * o p_{2}\right)\left\|\llbracket i n_{1} \rrbracket\left(o p_{1}\right)\right\| \llbracket i n_{2} \rrbracket\left(o p_{2}\right) \\
& \llbracket \bowtie \rrbracket_{n}\left(\text { in }_{1}, \text { in }_{2}, \text { out }_{1}\right) \quad=\quad \text { ! when } o p_{1} \bowtie o p_{2} \text { do tell }\left(o u t_{1}=1\right) \\
& \left.\| \text { when } \operatorname{not}\left(o p_{1} \bowtie o p_{2}\right) \text { do tell }\left(o u t_{1}=0\right)\right) \\
& \left\|\llbracket i n_{1} \rrbracket\left(o p_{1}\right)\right\| \llbracket i n_{2} \rrbracket\left(o p_{2}\right) \\
& \llbracket k \rrbracket_{n}\left(\text { out }_{1}\right)=\text { !tell }\left(\text { out }_{1}=k\right) \\
& \llbracket \text { select } 2 \rrbracket_{n}\left(\text { in }_{1}, \text { in }_{2}, \text { out }_{1}\right) \quad=\quad !\left(\text { when } o p_{1}=1 \text { do tell }\left(o u t_{1}=o p_{2,1}\right)\right. \\
& \left.\| \text { when } o p_{1}=0 \text { do tell }\left(o u t_{1}=o p_{2,2}\right)\right) \\
& \left\|\llbracket i n_{1} \rrbracket\left(o p_{1}\right)\right\| \llbracket i n_{2,1} \rrbracket\left(o p_{2,1}\right) \| \llbracket i n_{2,2} \rrbracket\left(o p_{2,2}\right) \\
& \llbracket \text { select } 3 \ldots \\
& \llbracket m e m \rrbracket_{n}\left(\text { in }_{1}, \text { out }_{1}\right) \quad=\quad ! \sum_{i \in[0, n]} \text { when } i=i n_{1} \text { do next tell }\left(o u t_{1}=i\right) \| \llbracket i n_{1} \rrbracket\left(o p_{1}\right) \\
& \text { Read-only table } \quad=\text { ? } \\
& \text { Read-write table } \quad=\text { ? } \\
& \llbracket @ \rrbracket_{n} \quad=\text { ? } \\
& \left.\llbracket \text { ffunction }(p, i, l) \rrbracket_{n}\left(\text { out }_{1}\right)=\text { !tell (ffunction }\left(p, i, l, \text { out }_{1}\right)\right)
\end{aligned}
$$

\subsubsection{GUI}

We suppose that the ambient sends signals label and $\neg$ label.

$\llbracket$ button ("label") $)\left(\right.$ out $\left._{1}\right)=$ when label do tell $\left(\right.$ out $\left._{1}=1\right)$

$\|$ when $\neg$ label do tell $\left(\right.$ out $\left._{1}=0\right)$

$\llbracket$ checkbox》

$\llbracket h s l i d e r \rrbracket=v$ slider $\ldots$

\subsection{Encoding composed signal processors}

\subsubsection{Sequential composition}

Example where $o_{A}<i_{B}$.

$\llbracket A: B \rrbracket\left(\right.$ in $_{1}$, in $_{2}$, in $_{3}$, out $\left._{1}\right)=$ local $z$ in in $\llbracket A \rrbracket\left(\right.$ in $_{1}$, in $_{2}$, zin $) \| \llbracket B \rrbracket\left(\right.$ in $_{3}, z i n$, out $\left._{1}\right)$

$A\left(\right.$ in $_{1}, \ldots$ in $_{n}$, out $_{1} \ldots$ out $\left._{m}\right): B\left(\right.$ in $_{1}, \ldots$ in $_{p}$, out $_{1} \ldots$ out $\left.\left._{q}\right)\right)$

Case $n<p$ :

$[A: B]\left(\right.$ in $_{1} \ldots$. in $_{n+p-m}$, out $\left._{1} \ldots o u t_{q}\right)=$ local $z i n_{1}, \ldots z i n_{m}$ in $[A]\left(\right.$ in $\left._{1} \ldots i n_{n}, z_{i n_{1} \ldots z i n_{m}}\right) \|[B]\left(z i n_{1}, \ldots z i n_{m}, i n_{n+1} \ldots i n_{n+}\right.$

Case $n=p$

$[A: B]\left(\right.$ in $_{1} \ldots$ in $_{n}$, out $_{1} \ldots$ out $\left._{q}\right)=$ local $z \operatorname{zin}_{1}, \ldots z \operatorname{zin}_{m}$ in $[A]\left(\right.$ in $\left._{1} \ldots \operatorname{in}_{n}, \operatorname{zin}_{1} \ldots z \operatorname{zin}_{m}\right) \|[B]\left(z \operatorname{in}_{1}, \ldots z\right.$ in $_{m}$, out $_{1} \ldots$ out $\left._{q}\right)$

Case $n>p$

$[A: B]\left(\right.$ in $_{1} \ldots$. in $_{n}$, out $_{1} \ldots$ out $\left._{q+m-p}\right)=$ local $z \operatorname{in}_{1}, \ldots z i n_{m}$ in $[A]\left(\operatorname{in}_{1} \ldots \operatorname{in}_{n}, \operatorname{zin}_{1} \ldots z \operatorname{in}_{m}\right) \|[B]\left(\operatorname{zin}_{1}, \ldots z \operatorname{zin}_{m}, \operatorname{in}_{m+1} \ldots i n_{q+}\right.$ 


\subsubsection{Parallel composition}

$A\left(\right.$ in $_{1}, \ldots$ in $_{n}$, out $_{1} \ldots$ out $\left._{m}\right), B\left(\right.$ in $_{1}, \ldots$ in $_{p}$, out $_{1} \ldots$ out $\left._{q}\right)$

$[A, B]\left(\right.$ in $_{1} \ldots$ in $_{n+p}$, out $_{1} \ldots$ out $\left._{m+q}\right)=[A]\left(\right.$ in $_{1} \ldots$ in $_{n}$, out $_{1} \ldots$ out $\left._{m}\right) \|[B]\left(\right.$ in $_{n+1} \ldots$ in $_{n+p}$, out $_{m} \ldots$ out $\left._{m+q}\right)$

\subsubsection{Split composition}

$$
\begin{aligned}
& \left.A\left(\text { in }_{1}, \ldots \text { in }_{n}, \text { out }_{1} \ldots \text { out }_{m}\right)<: B\left(\text { in }_{1}, \ldots \text { in }_{m . k}\right), \text { out }_{1} \ldots \text { out }_{q}\right)
\end{aligned}
$$

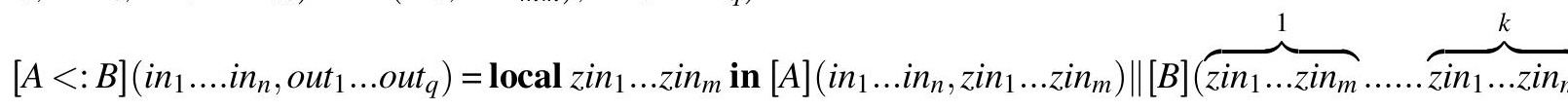

\subsubsection{Merge composition}

$A\left(\right.$ in $_{1}, \ldots$ in $_{n}$, out $_{1} \ldots$ out $\left._{m . k}\right):>B\left(\right.$ in $_{1}, \ldots$ in $_{m}$, out $_{1} \ldots$ out $\left._{q}\right)$

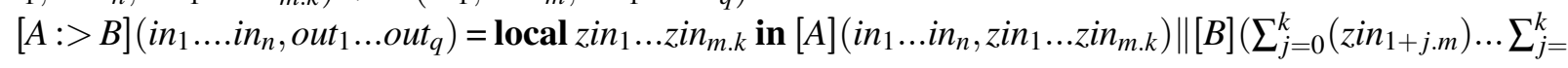

\subsubsection{Recursive composition}

$$
\left(A\left(\text { in }_{1}, \ldots \text { in }_{n+v}, \text { out }_{1} \ldots \text { out }_{m+u}\right) \sim B\left(\text { in }_{1}^{-1} \ldots . \text { in }_{u}^{-1}, \text { out }_{1} \ldots \text { out }_{v}\right)\right.
$$

$[A \sim B]\left(\right.$ in $_{1}, \ldots$ in $_{v} \ldots$ in $_{n+v}$, out $_{1} \ldots$ out $_{u} \ldots$ out $\left._{m+u}\right)=$ local $z$ in $_{1} \ldots z$ in $_{u}$ in $[A]\left(z\right.$ in $_{1} \ldots$ zin $_{u} \ldots$ out $_{1} \ldots$ out $\left._{n},\right) \|[B]\left(\right.$ in $_{1}$.

$\| \prod_{i \in[1, u]}$ tell $z \operatorname{zin}_{i}=0 \| ! \prod_{i \in[1, u]} \sum_{j \in[0, n]}$ when out $_{i}=j$ do next tell $\left(\operatorname{zin}_{i}=j\right)$

\subsubsection{Identity and cut box}

$\llbracket-\rrbracket\left(\right.$ in $_{1}$, out $\left._{1}\right)=\llbracket$ int $\rrbracket\left(\right.$ in $_{1}$, out $\left._{1}\right)=\llbracket$ float $\rrbracket\left(\right.$ in $_{1}$, out $\left._{1}\right)=$ !tell $\left(\right.$ out $_{1}=$ in $\left._{1}\right)$

$\llbracket ! \rrbracket\left(i n_{1}\right)=$ skip

\section{Example: The Karplus-Strong algorithm}

How can we make a difference in signals such as $i n_{1}$ and times. Is the out variable a parameter of the score? What about $i n_{1}$ ? The first one is a formal parameter, whereas the latter one is not.

\subsection{The noise generator}

times $\left(\right.$ in $_{1}$, timesout $\left._{1}\right)=[\times 110351245]\left(\right.$ in $_{1}$, timesout $\left._{1}\right)=$ local $z$ in $!$ tell $\left(\right.$ timesout $_{1}=$ $z \times$ in $\left._{1}\right) \| \llbracket 110351245 \rrbracket(z)$

plus $\left(\right.$ in $_{1}$, plusout $\left._{1}\right)=[(\times 110351245)+12345]\left(\right.$ in $_{1}$, plusout $\left._{1}\right)=$ local $z, y$ in !tell $\left(\right.$ plusout $_{1}=$ $z+y)\left\|\llbracket \times 110351245 \rrbracket\left(i n_{1}, z\right)\right\| \llbracket 12345 \rrbracket(y)$

random $($ outrandom $)=\llbracket \times(110351245)+12345 \sim \_\rrbracket($ outrandom $)=$ local $z, y$ in $[[(* 110351245)+12345]]\left(y\right.$, plusout $\left._{1}\right)\|[[-]](z, y)\| \|$ !tell $($ outrandom $=$ plusout $) \|$ tell $z=0 \| ! \sum_{j \in[0, n]}$ when plusout $=j$ do next tell $(z=j)$

division $($ divisionout $)=[1.0 / 2147483647.0]($ divisionout $)=\mathbf{l o c a l} z, y$ in $!$ tell $($ divisionout $=$ $z / y)\|\llbracket 1.0 \rrbracket(z)\| \llbracket 2147483647.0 \rrbracket(y)$ 
noise $($ outnoise $)=[$ random $*$ division $]($ outnoise $)=$ local $z, y$ in $!$ tell $($ outnoise $=$ $z * t)\|\operatorname{random}(z)\|$ division $(y)$

\subsection{The trigger}

$\operatorname{minus}(x$, minusout $)=[[x-\operatorname{mem}(x)]](x$, minusout $)=$ local $z$ in $!$ tell $($ minusout $=$ $x-z) \| \llbracket m e m(x) \rrbracket(z)$

impulse $(x$, impulseout $)=[(x-$ mem $(x))>0](x$, impulseout $)=$ local $z$ in $!$ tell $($ impulseout $=$ $z>0) \| \llbracket \operatorname{minus}(x, z) \rrbracket$

\section{Application to Interactive Scores}

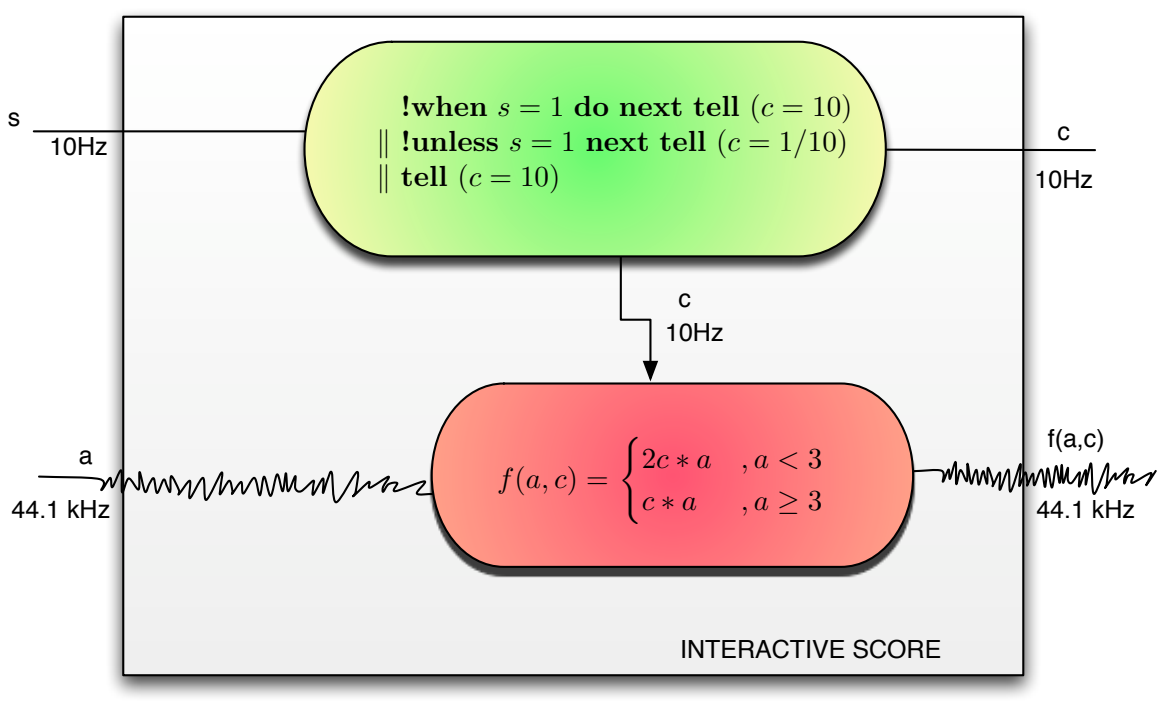

Figure 9: Example of Interactive Scores where Faust and Ntcc interact.

\section{References}

[1] A. Allombert, M. Desainte-Catherine, and M. Toro. Modeling temporal constrains for a system of interactive score. In G. Assayag and C. Truchet, editors, Constraint Programming in Music, chapter 1, pages 1-23. Wiley, 2011.

[2] J. Aranda, G. Assayag, C. Olarte, J. A. Pérez, C. Rueda, M. Toro, and F. D. Valencia. An overview of FORCES: an INRIA project on declarative formalisms for emergent systems. In P. M. Hill and D. S. Warren, editors, 
Logic Programming, 25th International Conference, ICLP 2009, Pasadena, CA, USA, July 14-17, 2009. Proceedings, volume 5649 of Lecture Notes in Computer Science, pages 509-513. Springer, 2009.

[3] J. D. A. Moreno, S. Passos, and M. Toro. On-line assembling mitochondrial DNA from de novo transcriptome. CoRR, abs/1706.02828, 2017.

[4] C. Olarte, C. Rueda, G. Sarria, M. Toro, and F. Valencia. Concurrent Constraints Models of Music Interaction. In G. Assayag and C. Truchet, editors, Constraint Programming in Music, chapter 6, pages 133-153. Wiley, Hoboken, NJ, USA., 2011.

[5] C. Patiño-Forero, M. Agudelo-Toro, and M. Toro. Planning system for deliveries in Medellín. ArXiv e-prints, Nov. 2016.

[6] A. Philippou and M. Toro. Process Ordering in a Process Calculus for Spatially-Explicit Ecological Models. In Proceedings of MOKMASD'13, LNCS 8368, pages 345-361. Springer, 2013.

[7] A. Philippou, M. Toro, and M. Antonaki. Simulation and Verification for a Process Calculus for Spatially-Explicit Ecological Models. Scientific Annals of Computer Science, 23(1):119-167, 2013.

[8] M. Raul, T. Mauricio, and C. Luz. Definicion de la arquitectura de referencia de un dominio: de la elucidacion al modelado. In R. Mazo, editor, Guia para la adopcion industrial de lineas de productos de software, pages 193-210. Editorial Eafit, 2018.

[9] J. M. C. Restrepo, A. F. Z. Palacio, and M. Toro. Assembling sequences of DNA using an on-line algorithm based on debruijn graphs. CoRR, abs/1705.05105, 2017.

[10] M. Toro. Exploring the possibilities and limitations of concurrent programming for multimedia interaction and graphical representations to solve musical csp's. Technical Report 2008-3, Ircam, Paris.(FRANCE), 2008.

[11] M. Toro. Probabilistic Extension to the Factor Oracle Model for Music Improvisation. Master's thesis, Pontificia Universidad Javeriana Cali, Colombia, 2009.

[12] M. Toro. Towards a correct and efficient implementation of simulation and verification tools for probabilistic ntcc. Technical report, Pontificia Universidad Javeriana, May 2009.

[13] M. Toro. Structured interactive musical scores. In M. V. Hermenegildo and T. Schaub, editors, Technical Communications of the 26th International Conference on Logic Programming, ICLP 2010, July 16-19, 2010, Edinburgh, Scotland, UK, volume 7 of LIPIcs, pages 300-302. Schloss Dagstuhl - Leibniz-Zentrum fuer Informatik, 2010. 
[14] M. Toro. Structured Interactive Scores: From a simple structural description of a multimedia scenario to a real-time capable implementation with formal semantics . PhD thesis, Univeristé de Bordeaux 1, France, 2012.

[15] M. Toro. Structured interactive music scores. CoRR, abs/1508.05559, 2015.

[16] M. Toro. Probabilistic Extension to the Concurrent Constraint Factor Oracle Model for Music Improvisation. ArXiv e-prints, Feb. 2016.

[17] M. Toro. Probabilistic Extension to the Concurrent Constraint Factor Oracle Model for Music Improvisation . Inteligencia Artificial, 57(19):37-73, 2016.

[18] M. Toro. CURRENT TRENDS AND FUTURE RESEARCH DIRECTIONS FOR INTERACTIVE MUSIC. Journal of Theoretical and Applied Information Technology, 69(16):5569-5606, 2018.

[19] M. Toro, C. Agón, G. Assayag, and C. Rueda. Ntccrt: A concurrent constraint framework for real-time interaction. In Proc. of ICMC '09, Montreal, Canada, 2009.

[20] M. Toro and M. Desainte-Catherine. Concurrent constraint conditional branching interactive scores. In Proc. of SMC '10, Barcelona, Spain, 2010.

[21] M. Toro, M. Desainte-Catherine, and P. Baltazar. A model for interactive scores with temporal constraints and conditional branching. In Proc. of Journées d'Informatique Musical (JIM) '10, May 2010.

[22] M. Toro, M. Desainte-Catherine, and J. Castet. An extension of interactive scores for multimedia scenarios with temporal relations for micro and macro controls. In Proc. of Sound and Music Computing (SMC) '12, Copenhagen, Denmark, July 2012.

[23] M. TORO, M. DESAINTE-CATHERINE, and J. CASTET. An extension of interactive scores for multimedia scenarios with temporal relations for micro and macro controls. European Journal of Scientific Research, 137(4):396409, 2016.

[24] M. Toro, M. Desainte-Catherine, and C. Rueda. Formal semantics for interactive music scores: a framework to design, specify properties and execute interactive scenarios. Journal of Mathematics and Music, 8(1):93-112, 2014.

[25] M. Toro, A. Philippou, S. Arboleda, M. Puerta, and C. M. Vélez S. Meanfield semantics for a process calculus for spatially-explicit ecological models. In C. A. Muñoz and J. A. Pérez, editors, Proceedings of the Eleventh International Workshop on Developments in Computational Models, Cali, Colombia, October 28, 2015, volume 204 of Electronic Proceedings in Theoretical Computer Science, pages 79-94. Open Publishing Association, 2016. 
[26] M. Toro, A. Philippou, C. Kassara, and S. Sfenthourakis. Synchronous parallel composition in a process calculus for ecological models. In G. Ciobanu and D. Méry, editors, Proceedings of the 11th International Colloquium on Theoretical Aspects of Computing - ICTAC 2014, Bucharest, Romania, September 17-19, volume 8687 of Lecture Notes in Computer Science, pages 424-441. Springer, 2014.

[27] M. TORO, C. RUEDA, C. AGÓN, and G. ASSAYAG. Ntccrt: A concurrent constraint framework for soft real-time music interaction. Journal of Theoretical \& Applied Information Technology, 82(1), 2015.

[28] M. TORO, C. RUEDA, C. AGÓN, and G. ASSAYAG. Gelisp: A framework to represent musical constraint satisfaction problems and search strategies. Journal of Theoretical \& Applied Information Technology, 86(2), 2016. 